\title{
Wave Interaction in Oxygen Magnetoplasmas
}

\author{
K. V. Narasinga Rao \\ Air Force Cambridge Research Laboratories, Bedford, Mass. \\ (Received October 22, 1964: revised November 27, 1964)
}

\begin{abstract}
Electron cyclotron resonance absorption of microwaves in decaying oxygen magnetoplasmas is utilized to alter the plasma parameters such as the "temperature" and number density of the electrons. The technique of cross modulation of microwaves in partially filled plasma waveguides is used in this investigation. It is shown that for small amplitudes $(\sim 120 \mathrm{~mW}$ at $5.3 \mathrm{Gc} / \mathrm{s})$ of the resonant heating signal the inelastic collision of low energy electrons introduce a barrier for rapid heating the electron gas. For higher incident amplitudes $(\sim 500 \mathrm{~mW}$ at $5.3 \mathrm{Gc} / \mathrm{s})$, not only does rapid heating of the electron gas occur, but also additional electron production and loss mechanisms are observed. The implications of these results on (a) apparent discrepancies in measurements of attachment coefficient by microwave methods, and (b) control of ionospheric plasma parameters by radio wave absorption are discussed.
\end{abstract}

\section{Introduction}

Irradiation of the earth's ionosphere by radio waves of appropriate frequency and amplitude has been suggested as a means of controlling the "temperature" and number density of electrons by Bailey and Goldstein [1958] and recently by Molmud [1963]. The familiar "Luxemberg effect" or interaction of two radio waves of different frequencies in a specific region of the ionosphere [Tellegan 1933] and cross modulation of microwaves in gaseous plasmas produced in the laboratory [Goldstein, et al., 1953] are examples of such a control of plasma parameters. The effects produced in a magnetoplasma when one of the interacting microwaves is of a frequency equal to the cyclotron frequency of the electrons has been investigated by the author and others at the University of Illinois [Narasinga Rao et al., 1961]. Results from these experiments, performed in low pressure, decaying neon magnetoplasmas have shown that the expected transfer of microwave energy to the electron gas was rapid; which in turn reduced magnetic confinement of the plasma and altered the propagation characteristics of microwaves.

Similar experiments performed in atmospheric gaseous magneto plasmas $\left(\mathrm{N}_{2}\right.$ and $\left.\mathrm{O}_{2}\right)$ demonstrated [Narasinga Rao and Goldstein, 1963a] that inelastic collisions of low energy electrons with neutral particles constitute an efficient barrier for the rapid heating of the electron gas. To overcome this barrier and transfer resonant electromagnetic energy to the electron gas it was suggested [Narasinga Rao and Goldstein, 1963a] that higher incident microwave electric field intensities and/or lower pressures were necessary. Experimental results on wave interaction in oxygen magnetoplasmas (reported in this paper) indicated that in a 0.2 torr oxygen magneto plasma absorption of resonant microwaves $(\sim 5 \mathrm{Gc} / \mathrm{s}$, and of an amplitude $\sim 1 \mathrm{~W})$ not only altered the electron loss rate but also enhanced the electron production rate in a controllable manner. However, the visible light intensity emanating from the heated plasma showed that the maximum interaction of the resonant microwaves with the plasma was restricted to a small spatial region of the plasma column, resulting in large gradients of $\mathrm{N}_{e}$ and $\mathrm{T}_{e}$. Similar effects are predicted even for inhomogeneous, isotropic plasmas illuminated with high power electromagnetic waves [Papa, July 1964, private communication]. Numerically computed solutions to the Boltzmann equation giving the electron energy distribution in slightly ionized air under the influence of electric and magnetic fields have been obtained by Carleton and Megill [1962]. A rather comprehensive survey covering the contributions made by workers in the United States during 1960-1963 on the topic of "Electromagnetic Wave Propagation in Inhomogeneous Plasmas and/or Magneto-Plasmas" has also been made by Hoffman [1964] recently.

We proceed in the next section to discuss briefly the rates of electron removal and production mechanisms in the oxygen magnetoplasmas and their dependence on electron energy. Also included in this section are considerations on the electromagnetic energy transfer to the electron gas under cyclotron resonance conditions. The experimental technique of wave interaction in magneto plasmas and the results obtained are described in section 3 . We then conclude by a discussion of these results and their application to the problem of altering the plasma parameters by radio wave absorption in the ionosphere. 


\section{Electron Density Decay and Electron Heating in Afterglows}

\subsection{Electron Removal and Production Mechanisms in Low Pressure Oxygen Magnetoplasmas}

As is well known the principal electron removal processes in a gaseous discharge plasma are (1) diffusion to the boundaries and subsequent "wall recombination," (2) volume recombination of electrons with positive ions and (3) attachment to electronegative molecules forming negative ions. A detailed discussion on these processes (a single dominating loss process or a combination of two different processes) is given by Oskam [1958] and will not be elaborated here. For the conditions used in the present experiments, i.e., in a low pressure $(\leqslant 0.2$ torr) weakly icnized (degree of ionization $\delta \leqslant 10^{-5}$ ), oxygen magnetoplasma $(\mathrm{H} \sim 2 \mathrm{kG})$ it is reasonable to assume that the dominant electron removal process is by attachment. Using similar experimental conditions, Anisimov and others [1964] have shown that the electron attachment frequency is $(9 \pm 1) \times 10^{4} /$ torr/sec and the volume recombination coefficient is $(1.90 \pm 0.1) \times 10^{-7} \mathrm{~cm}^{3} / \mathrm{sec}$. Since one of the objects of our experiment is to demonstrate that resonance absorption of microwave energy by the electron gas can efficiently alter the prevailing electron density in a decaying plasma, we consider in this section the energy dependence of electron attachment coefficients for oxygen.

Several investigators have studied the attachment process in oxygen and the dependence of attachment coefficient on electron energy in the range from few hundredths to few electron volts. At oxygen pressures of the order of tens of $\mathrm{mm} \mathrm{Hg}$, and at low electron energies $(<1 \mathrm{eV})$ it was concluded by Chanin et al., [1962] that the attachment process involves the three-body process. However at the oxygen pressure used in our experiment $(\leqslant 0.2$ torr) the decay of electron density is assumed to be mainly by electron attachment involving a two-body process. This can be justified by considering the time rate of change of electron density represented as

$$
\frac{d N_{e}}{d t}=-\beta N\left(0_{2}\right) N_{e}-K N^{2}\left(0_{2}\right) N_{e}
$$

where $N_{e}, N$, are respectively the concentrations of electrons and neutral oxygen molecules, $\beta$ is the twobody and $K$ the three-body attachment coefficient. For electron energies $\sim .04 \mathrm{eV}$ and at neutral oxygen pressures $\leqslant 0.2$ torr, the electron density decay by three-body attachment processes is at least an order of magnitude slower than that by two body attachment processes, e.g., at .04 eV, $\beta \sim 3 \times 10^{-12} \mathrm{~cm}^{3} / \mathrm{sec}$ [Anisimov et al., 1964] and $K \sim 3 \times 10^{-30} \mathrm{~cm}^{6} / \mathrm{sec}$ [Chanin et al., 1962].

The variation of the two-body attachment coefficient, $\beta$, with electron energy is shown in figure 1 , as curves $\mathrm{a}$ and $\mathrm{b}$. Curve $\mathrm{a}$ is obtained from the data of probability of attachment " $h$ " measured by Bradbury [1933] and that of momentum transfer collision frequency measured by Mentzoni [1964]. Curve b is obtained from the data of "swarm" experiments of Chanin [1959]. Thus, it is apparent that when electron energy in a low pressure decaying oxygen magnetoplasma is increased from $.04 \mathrm{eV}$ to a few volts (as is the case in our experiments) it can be seen that the rate of attachment decreases first and then increases. However, for electron energies $\geqslant 0.2 \mathrm{eV}$, electron-neutral collisions produce appreciable number of vibrationally excited molecules whose collisions with existing negative ions could constitute a mechanism for producing new electrons by the reaction

$$
0_{2}^{-}+0_{2}^{*} \longrightarrow 0_{2}+0_{2}+e
$$

as suggested by Craggs [1957]. For electron energies attained in our experiment this process of production of new electrons appears to be more favorable than that by direct ionization since the ionization cross section is small [Bates 1962].

\subsection{Resonance Ábsorption of Microwaves and Enhancement of Electronic Energy}

In this section we consider briefly the gain in electronic energy by resonance absorption of microwave power and the energy lost by electrons in elastic and in inelastic collisions. The time rate of change of electron energy may be expressed as

$$
\begin{aligned}
\frac{d W_{e}}{d t}=\left.\frac{d W_{e}}{d t}\right|_{\text {source }}-\frac{2 m}{M} \nu_{e m}\left(W_{e}-W_{0}\right) & \\
& -\lambda \nu_{i n}\left(W_{e}-W_{0}\right),
\end{aligned}
$$

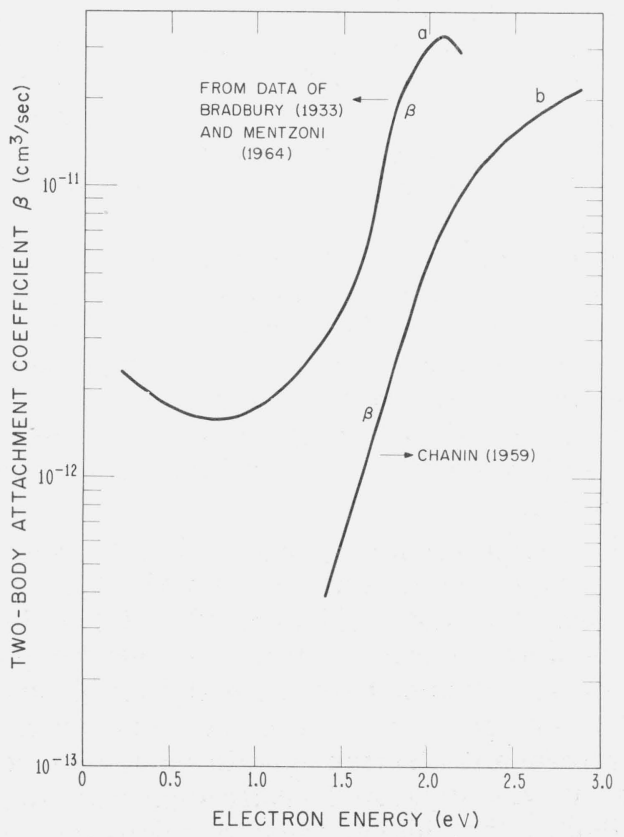

Figure 1 . 
where the first term on the right hand side of this equation represents the energy gained by electrons due to absorption of microwave energy and the second and third terms represent the energy lost in elastic and in inelastic collisions. In (3) $2 m / M$ is the classical $G$-factor, $\nu_{i n}$ is the inelastic collision frequency, and $\lambda$ the fractional energy loss in an inelastic collision. The energy gain term may be simplified [Narasinga Rao and Goldstein, 1963a]

$$
\frac{d W_{e}}{d t} \mid \simeq \text { source } \frac{N_{e} e^{2} E^{2}}{m \nu_{e m}} .
$$

In (4) the assumption is made that when $\omega_{H}=\omega$ and $\nu_{e m}^{2} \ll \omega^{2}$ the real part of the main diagonal component $\left(\sigma_{x x}\right)$ of the complex conductivity tensor may be expressed as

$$
\operatorname{Re} \sigma_{x x}=\frac{N_{e} e^{2}}{m \nu_{e m}}
$$

Thus, it is apparent that the time rate of change of energy by cyclotron resonance absorption may be increased by utilizing high electric fields and/or low pressures. However, the collision frequency also rises, since it is proportional to energy [Chanin et al., 1962; Mentzoni, 1964] and thus the absorption of resonant microwave energy decreases when electron energy is increased. In addition, the energy lost in elastic and inelastic collisions even at low electron energies $(<0.2 \mathrm{eV})$ may impede the transfer of electromagnetic energy to electron gas for small resonant electric field intensities as observed earlier [Narasinga Rao and Goldstein, 1963b].

\section{Experimental Technique and Results}

\subsection{Technique}

The technique of cross modulation of two microwave signals guided through a waveguide (WR 187) containing the oxygen magnetoplasmas is used to study the phenomena resulting from the cyclotron resonance absorption of a microwave signal by the electron gas. The detailed description of the apparatus has been given by us earlier [Narasinga Rao et al., 1961]. The microwave signal which is used as a heating signal is of a frequency equal to 5.30 $\mathrm{Gc} / \mathrm{s}$ and of a maximum amplitude of $1000 \mathrm{~mW}$. The sensing microwave signal is of a frequency. equal to $5.45 \mathrm{Gc} / \mathrm{s}$ and of a maximum amplitude of $50 \mu \mathrm{W}$. The external d-c magnetic field is adjusted so that the electron cyclotron resonance prevails, i.e., $f_{H}$ $=\frac{e B}{2 \pi m}=f_{H W}=5.30 \mathrm{Gc} / \mathrm{s}$. The decaying magnetoplasma is produced in a cylindrical discharge tube, (inner radius $7 \mathrm{~mm}$ and $12 \mathrm{~cm}$ long) by means of a $2 \mu$ sec wide, $4 \mathrm{kV}$ pulse at a repetition rate of 500 pps. By appropriate timè sequencing of the pulsed heating and sensing microwave signals, with reference to the d-c breakdown pulse, and measuring their transmission characteristics it is possible to disturb and probe the state of the decaying magnetoplasma. In particular we report here the results obtained when the heating signal input amplitude is varied from 30 $\mathrm{mW}$ to $1000 \mathrm{~mW}$.

\subsection{Results}

Typical oscillograms representing the wave interaction phenomena and emission of visible light intensity from a heated oxygen magnetoplasma are shown in figure 2. Traces 1 and 3 in this figure are the transmitted amplitudes of the heating and sensing microwave signals without the plasma in the waveguide. The time is increasing from left to right and the leading edge of the heating microwave pulse is incident on the plasma column $1 \mathrm{msec}$ after the termination of the d-c breakdown pulse. Trace 2 is the amplitude of the heating pulse transmitted through the magnetoplasma (for resonance condition $f_{H W}=f_{H}$ ). It is noted that the leading edge of this pulse is attenuated almost completely (reflected amplitude was negligible) due to resonance absorption followed by increased transmission and approximately after $4 \mu$ sec, the transmitted amplitude decreases again, indicating an increase in the electron number density. Oscillograms 4 and 5 represent the transmitted amplitudes of the pulsed sensing microwave signals through the magnetoplasma without and with the incidence of the heating microwave signal. The gradual rise in transmission of the sensing microwave signal (trace 4), is due to the slow decay of the electron density. In

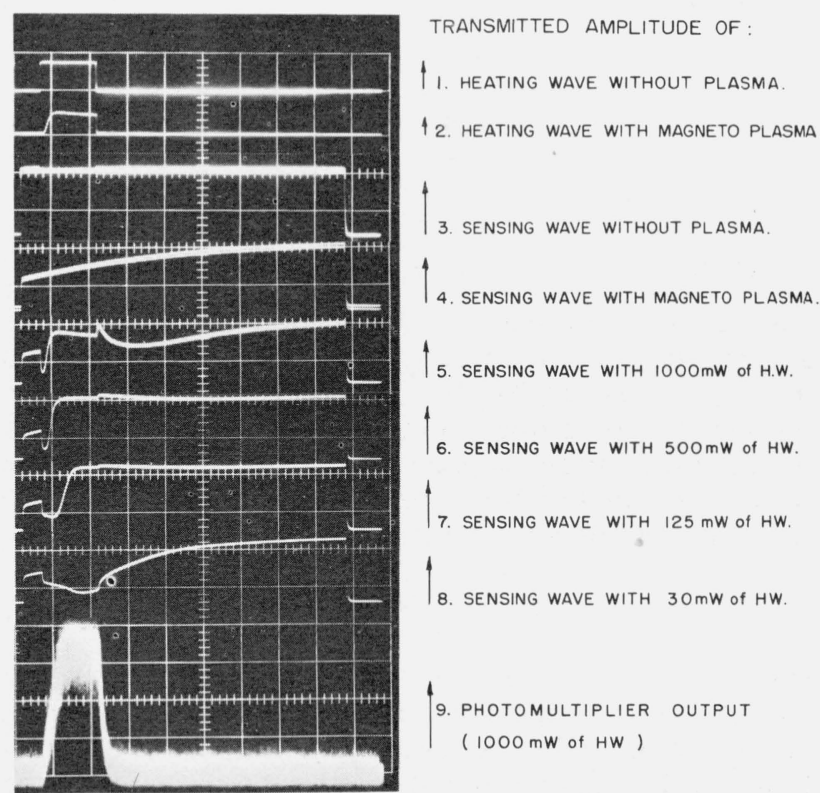

WAVE INTERACTION IN $\mathrm{O}_{2}$ MAGNETO PLASMA; $p=0.2$ TORR.,

HEATING WAVE $(H W)$ FREQUENCY $=5.30 \mathrm{Gc}$., DC MAGNETIC FIELD $=1.88 \mathrm{kG}$, SENSING WAVE FREQUENCY $=5.45 \mathrm{Gc}$.,

TIME SCALE $=20 \mu \mathrm{sec} / \mathrm{cm}$

FiguRE 2 


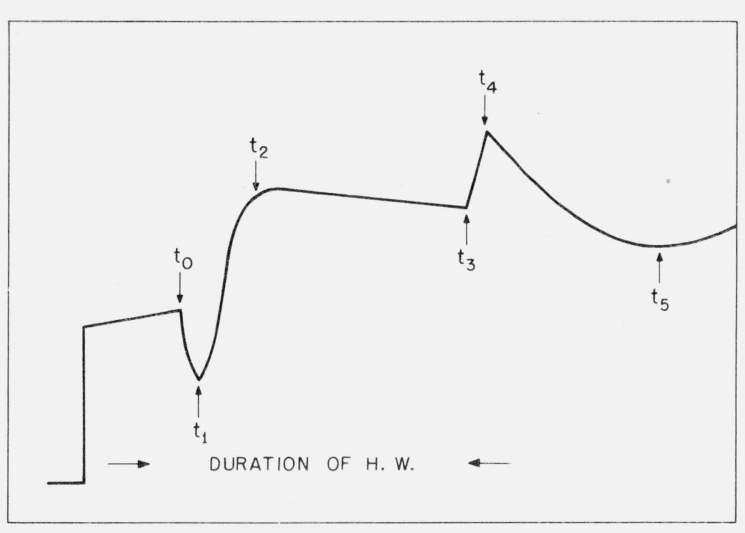

SKETCH OF A PORTION OF TRACE 5 OF FIG. 2

FIGURE 3.

trace 5 the gyromodulation of the sensing microwave signal during and in the wake of the $1000 \mathrm{~mW}$ pulsed heating microwave signal is seen. For clarity's sake, the initial portion of this sensing microwave signal amplitude is sketched in figure 3 . Immediately following the initiation of the heating pulse, the absorption of the sensing microwave signal increases and reaches a maximum at instant $t_{1}$. This maximum absorption of the sensing microwave signal occurs since the real part of the conductivity $\left(\sigma_{x x s}\right)$ as "seen" by the sensing microwave signal attains a maximum value when the electronic collision frequency is approximately given by the relation [Narasinga Rao et al., 1961]

$$
\frac{\nu_{e m}}{\omega_{S}} \simeq\left|1-\frac{\omega_{H}}{\omega_{S}}\right|
$$

During the period $\left(t_{2}-t_{1}\right)$ the decrease in attenuation of the sensing microwave signal is caused by the increase in the attachment rate and/or since $\frac{\nu}{\omega_{S}}>\left|1-\frac{\omega_{H}}{\omega_{S}}\right|$. The gradual increase in absorption from $t_{2}$ to $t_{3}$ is attributed to an increase in $N_{e}$. This rise in electron density is caused by the reaction (2). As mentioned earlier, the electron energies here are not sufficient to produce direct ionization. The electron energy may be estimated from the value of $\nu_{e m}$ at $t_{1}$, from (6) and the relationship given by Mentzoni [1964], i.e.,

$$
\nu_{e m}=4 \cdot 8 \times 10^{-8} \times N\left(O_{2}\right) \times W_{e}\left(\sec ^{-1}\right)
$$

where $N\left(\mathrm{O}_{2}\right)=$ number density of oxygen molecules and $W_{e}$ is the electron energy in $\mathrm{eV}$. The electron energy estimated thus is approximately $3 \mathrm{eV}$. Limitations on this method of estimating the electron energy are described in the next section. After the termination of the heating pulse, i.e., after instant $t_{3}$, the electron gas tends to cool both by inelastic and elastic collisions. The decrease in absorption during $t_{4}-t_{3}$ is accounted for by a rapid decrease in $N_{e}$. However, it should be pointed out that during the interval $t_{5}-t_{3}$, the electronic collision frequency still satisfies the condition that $\frac{\nu}{\omega_{S}}>\left|1-\frac{\omega_{H}}{\omega_{S}}\right|$ and at instant $t_{5}$ the condition $\frac{\nu}{\omega_{S}} \simeq\left|1-\frac{\omega_{H}}{\omega_{S}}\right|$ is once again realized. This is similar to the wave-interaction phenomena in neon magnetoplasma at high pressures [Narasinga Rao and Goldstein, 1963b]. The relaxation of the transmitted amplitude to the unattenuated value after instant $t_{5}$ is both due to the decrease in $N_{e}$ and cooling of the electron gas. It should be mentioned that when this high amplitude $(1000 \mathrm{~mW})$ of the heating microwave signal is utilized, the photomultiplier output indicated that there is detectable visible intensity at the front portion of the plasma (trace 9). Measurements on the visible light intensity along the plasma column showed that the light intensity decreased by a factor of 5 within $2 \mathrm{~cm}$ of the microwave transmission path. Thus, as explained before for wave interaction in neon magnetoplasma [Narasinga Rao and Goldstein, 1963b], it was concluded that the high power resonant microwave signal was interacting efficiently only in a small region of the oxygen plasma column.

Traces 6, 7, and 8 of (2) show the wave interaction phenomena for 500,125 , and $30 \mathrm{~mW}$ of heating microwave signals. When the amplitudes of the heating microwave signal is decreased, as expected, the rate of the heating was slower which was inferred from the observation that the first maximum absorption [e.g., instant $t_{1}$ in (3)] occurs at later instants, i.e., instant $t_{1}$ moves to the right as the amplitude of the heating signal is decreased. For moderately low amplitudes of the heating signal $(<125 \mathrm{~mW})$ the relatively constant attenuation periods of the sensing microwave signal during the heating pulse demonstrates that inelastic collisions of low energy electrons constitute a barrier for the rapid transfer of resonant microwave energy to the electron gas. Such quasi-steady states of the plasma (as seen by constant attenuation periods of the sensing microwave signal) were not observed on this time scale for the higher amplitude heating signals (traces 5 and 6 ).

\section{Discussion of Results}

The enhancement in the momentum transfer collision frequency and therefore the electron energy, was inferred from the observed wave interaction phenomena in magnetoplasmas subjected to resonance absorption in microwave power. The available experimental data on the energy dependence of the momentum transfer collision frequency, from $0.04 \mathrm{eV}$ to $\sim 3 \mathrm{eV}$ are limited to only small ranges [Phelps 1960; Gilardini 1963; and Mentzoni 1964], and are determined by different techniques. It should be pointed out that in addition to the lack of the experimental evidence for the exact energy dependence of the electronic collision frequency, the interpretation of wave interaction is based on the assumption that the plasma is in a steady state and has uniform characteristics over the entire spatial region of the plasma. 
This assumption is certainly not justified when the high power resonant microwaves are used. How ever, from the consistent variation of the gyromodulation phenomenon with input power of the heating signal (e.g., movement of the maximum absorption instant, $t_{1}$ in fig. 3 ) leads us to the interpretation that the enhanced collision frequency is not far from the value given by (6).

The increased absorption of the sensing microwave signal observed during the time interval $t_{3}-t_{2}$ is interpreted as that caused by an increase in $N_{e}$. It should be noted that since $\sigma_{x x s} \propto N_{e} / \nu_{e m}$ during this period, the increased absorption may also be attributed to a decrease in the collision frequency. This interpretation is possible if the momentum transfer collision cross section decreases with increasing electron energy in the range of interest. Such a variation has not been observed [Gilardini, 1963].

The observation that the transmitted sensing wave amplitude returns to its unattenuated value faster after the termination of low amplitude heating pulses is further evidence that enhancement of the electron attachment rate occurs even during the application of the heating pulse.

Thus, if sufficient numbers of vibrationally excited molecules survive for long intervals of time in afterglow plasmas, the collisions between these excited molecules and negative ions could constitute a mechanism for detachment of electrons and may lead to erroneous values for attachment coefficients inferred from measurements of $d N_{e} / d t$ by microwave methods.

\section{Conclusions}

In conclusion the wave interaction phenomena observed in oxygen magnetoplasmas at low pressures indicated that:

(1) Cyclotron resonance absorption of moderately low amplitude microwaves $(\sim 100 \mathrm{~mW})$ enhances the electron energy sufficiently to increase the prevailing two-body attachment rate of electrons to neutral oxygen molecules.

(2) Absorption at higher amplitudes $(<1000 \mathrm{~mW})$ of the resonant microwave signals enhances the electron energy rapidly, and provides a mechanism for detachment of electrons from negative ions.

(3) The visible light intensity emitted by the "heated" magnetoplasma showed that the maximum interaction between the resonant microwave signal and the plasma occurs only in a small region.

\section{Applications}

The technique of wave interaction of electromagnetic waves in the ionospheric layers (altitudes $<100 \mathrm{~km}$ ) may be used to determine several rate coefficients. In particular if the resonant signal is transmitted from a rocket [Hodges, 1964], intense amplitudes of this signal cause large disturbances (in electron energy and number density) only in a small region which are detectable by the nonresonant sensing signal. That the depth of penetration of the resonant signal is limited to only a small region has been mentioned by Ginzburg [1961] and verified in our experiments indirectly. However, even moderately low amplitudes of the resonant signal are sufficient to enhance the electron energy to cause an enhancement in the two-body attachment rate.

It is of interest to mention that several characteristics of the hot dense plasma produced by the interaction of powerful $(\sim 50 \mathrm{~kW})$ resonant microwaves $(\sim 3 \mathrm{Gc} / \mathrm{s})$ with low pressure $\left(<10^{-3}\right.$ torr $)$ air are also being studied currently by Fessenden [1964] at M.I.T.

The author acknowledges the many valuable discussions he had with L. Goldstein of the University of Illinois and the assistances and cooperation of the members of the Microwave Physics Laboratory of AFCRL where this work was performed.

\section{Appendix. List of Symbols}

$N_{e}=$ electron density,

$H=$ d-c magnetic field intensity,

$N=$ number density of oxygen molecules,

$\beta=$ two-body attachment coefficient,

$K=$ three-body attachment coefficient,

$W_{e}=$ mean electron energy,

$m, M=$ mass of electron and oxygen molecule,

$e=$ charge of an electron,

$\nu_{e m}=$ momentum transfer collision frequency of electrons with molecules,

$\nu_{\text {in }}=$ inelastic collision frequency,

$\lambda=$ fractional energy lost in an inelastic collision,

$E=$ incident microwave electric field intensity,

$f_{H}=$ cyclotron frequency of electrons,

$f_{H W}=$ frequency of "heating" wave (resonant signal),

$f_{S}=$ frequency of "sensing" wave,

$\sigma_{x x}=$ main diagonal component of the conductivity tensor,

$\sigma_{x x s}=$ main diagonal component of the conductivity as "seen" by sensing wave.

\section{References}

Anisimov, A. I., N. I. Vinogradov and V. E. Golant (1964), Measurement of volume removal coefficient for electrons in plasma decay in oxygen, Zhur. Tekn. Fiz. 33, No. 9, Sept. 1963, English Translation: Sov. Physics Tech. Phy. 8, 850, (1964).

Bailey, V. A. and L. Goldstein (1958), Control of ionosphere by means of radio waves, J. Atmospheric and Terrest. Phy. 12, 216.

Bates, D. R. (1962), Atomic and molecular processes, p. 240 (Academic Press, New York, N.Y.).

Bradbury, N. E. (1933), Electron attachment and negative ion formation in oxygen and oxygen mixtures, Phy. Rev. 44, 883.

Carleton, N. P., and L. R. Megill (June 15, 1962), Electron energy distribution in slightly ionized air under the influence of electric and magnetic fields. Phys. Rev. 126, 2089-2099.

Chanin, L. M., A. V. Phelps, and M. A. Biondi (1959), Measurement of the attachment of slow electrons in oxygen, Phy. Rev. Letters 2, 344 .

Chanin, L. M., A. V. Phelps, and M. A. Biondi (1962), Measurement of the attachment of low energy electrons to oxygen molecules, Phy. Rev. 128, 219-230. 
Craggs, J. D. (Oct. 1957), Some experiments on negative ions in gases, Proc. of III Conf. on Ionization Phenomenon in Gases, pp. 203-207, Conference held in June 1957 (The Italian Physical Society, Venice, Italy).

Fessenden, T. J., (July 15, 1964), Electron cyclotron resonance discharge MIT Quarterly Prog. Rpt. 14, Res. Lab. of Electronics, Cambridge, Mass.

Gilardini, A. (May 1963), Microwave determinations of afterglow temperatures and electron collision parameters in nitrogen and oxygen, Tech. Report AF61(052)-39.

Ginzburg, V. L. (1961), Propagation of electromagnetic waves in plasma, translated from the Russian by Royer and Roger. eds. W. L. Sadowski, and D. M. Gallik (Gordon and Breach Science Publishers, Inc., New York, N.Y.)

Goldstein, L., J. M. Anderson, and G. Clark (1953), Interaction of microwaves propagated through a gaseous discharge plasma, Phy. Rev. Letter 90, 151

Hodges, R. L. (1964), Gyro-interaction rocket experiments in the lower ionosphere, Ph. D. Thesis, Dept. of Elect. Eng. Univ. of Illinois, Urbana, Ill.

Hoffman, W. C. (April 1964), Electromagnetic wave propagation in inhomogeneous plasmas and/or magnetoplasmas, Radio Sci. J. Res. NBS/USNC-URSI 68D, No. 4, 486-489.
Mentzoni, M. H. (1964), Electron momentum transfer collisions in oxygen for thermal electrons, Radio Sci. J. Res. NBS 69D, No. 2, 211-215.

Molmud, P. (1963), Altering the Electron Density in the $D$ Region: A New Tool for Ionosphere D-Layer Research, J. Geophys. Res. 68, No. 19, 5443 .

Narasinga Rao, K. V., and L. Goldstein (1963a), Gyro interaction of microwaves in atmospheric gaseous magnetoplasmas, Proc. of VI Conference Internationale sur les Phenomenes d'Ionization dans les Gaz (S.E.R.M.A.), Paris, 1963.

Narasinga Rao, K. V., and L. Goldstein (1963b), Gyro interaction of microwaves in noble gaseous magnetoplasmas, Proc. of VI Conference Internationale sur les Phenomenes d'Ionization dans les Gaz(S.E.R.M.A.), Paris, 1963.

Narasinga Rao, K. V., J. T. Verdeyen, and L. Goldstein (1961), Interaction of microwaves in gaseous plasmas immersed in magnetic fields, Proc. of IRE 49, No. 12, 1877-1899.

Oskam, H. J. (Aug. 1958), Microwave investigation of disintegrating gaseous discharge plasmas, Philips Res. Rept. 13, 335-400.

Phelps, A. V. (1960), Propagation constants for electromagnetic waves in weakly ionized dry air, J. Appl. Phys. 31, 1723.

Tellegen, B. D. H. (1933), Interaction between radio waves? Nature $131,840,1933$.

(Paper 69D4-496) 